Case Study

\title{
Effects of a novel forced intensive strengthening technique on muscle size and upper extremity function in a patient with chronic stroke
}

\author{
Hee-won Jeong, BS, PT ${ }^{1)}$, Seung-chul Chon, PhD, $\mathrm{PT}^{1)^{*}}$ \\ 1) Department of Physical Therapy, Konyang University: Gwanjeo-Dong, Seo-Gu, Daejeon 302-718, \\ Republic of Korea
}

\begin{abstract}
Purpose] This research demonstrated a forced intensive strength technique as a novel treatment for muscle power and function in the affected upper extremity muscle to determine the clinical feasibility with respect to upper extremity performance in a stroke hemiparesis. [Subject and Methods] The subject was a patient with chronic stroke who was dependent on others for performing the functional activities of his affected upper extremity. The technique incorporates a comprehensive approach of forced, intensive, and strength-inducing activities to enhance morphological changes associated with motor learning of the upper extremity. The forced intensive strength technique consisted of a 6-week course of sessions lasting 60 minutes per day, five times a week. [Results] After the 6 -week intervention, the difference between relaxation and contraction of the affected extensor carpi radialis muscle increased from 0.28 to $0.63 \mathrm{~cm}^{2}$, and that of the affected triceps brachii muscle increased from 0.30 to $0.90 \mathrm{~cm}^{2}$. The results of clinical tests including the modified Ashworth scale (MAS; from 1+ to 1), muscle strength (from 15 to $32 \mathrm{~kg}$ ), the manual function test (MFT; scores of 16/32 to 27/32 score), the Fugl-Meyer assessment (FMA; scores of 29/66 to 49/66 score), and the Jebsen-Taylor hand function test (JTHFT; from 38/60 to 19/60 sec) were improved. [Conclusion] Our results suggest that the forced intensive strength technique may have a beneficial effect on the muscle size of the upper extremity and motor function in patients with chronic stroke.

Key words: Upper extremity, Strength, Stroke
\end{abstract}

(This article was submitted Aug. 4, 2015, and was accepted Aug. 19, 2015)

\section{INTRODUCTION}

Loss and impairment of sensorimotor function is common after a stroke, and restoration of sensorimotor function is a major treatment goal for patients with stroke hemiparesis ${ }^{1)}$. This is especially important in the upper extremity (UE), where the function has been shown to persist after the initial stroke in only $50 \%$ of stroke survivors ${ }^{2}$. Therefore, considerable rehabilitative effort is required to maximize voluntary muscle control, and regaining UE function depends on attempting to use the involved extremity as well as managing compensatory use of the noninvolved limb.

There does not appear to be a clear consensus on the best therapeutic method for restoring UE sensorimotor function, as various studies have reported different results ${ }^{1-3)}$. Thus, a number of neurodynamic approaches have been developed based on weight bearing ${ }^{4}$, the forced use approach ${ }^{5)}$, repetitive practice ${ }^{6)}$, bilateral training ${ }^{7)}$, and motor function using a functional electrical stimulator $(\mathrm{FES})^{8}$. All of these

*Corresponding author. Seung-chul Chon (E-mail: keyjune@ konyang.ac.kr)

C 2015 The Society of Physical Therapy Science. Published by IPEC Inc. This is an open-access article distributed under the terms of the Creative Commons Attribution Non-Commercial No Derivatives (by-ncnd) License $<$ http://creativecommons.org/licenses/by-nc-nd/3.0/> . methods have been implicated in reestablishment of motor control and relearning by optimizing motor recovery in stroke patients.

To address inconsistencies associated with past rehabilitative research, the forced intensive strengthening technique (FIST) was developed ${ }^{4-8)}$. This technique incorporates a combination of highly forced, intensive, and strengthinducing activities to enhance morphological changes associated with motor learning of the $\mathrm{UE}^{4-6)}$. A comprehensive approach, such as the FIST, potentially confounds the effects of the function and strengthening of the UE.

Although multidisciplinary techniques such as the FIST may be consistently implemented between physiotherapy centers, the effectiveness of these approaches has not been objectively explored. As a result, the underlying mechanism supporting an intervention such as the FIST has not been quantified. Furthermore, the use of real-time ultrasound imaging (USI) has been advocated to determine morphological changes that are representative of muscle strength or weakness and associated with muscular exercises ${ }^{9)}$. Therefore, the purpose of this pilot study was to quantitatively investigate the effect of the FIST on clinical measures and muscle architecture immediately before and after an intervention program in a poststroke patient. 


\section{SEBJECT AND METHODS}

The participant was a 56-year-old, left-handed man with hemiparesis who had been diagnosed with middle cerebral artery infarction by functional MRI. The density of the hemorrhagic infarction area, which included the right frontal insular cortex and the area near the temporal-parietal-occipital junction, was decreased due to edema. In addition, the left cerebellar tonsil and hemisphere showed old multifocal infarctions. The patient had experienced an ischemic stroke one year before enrollment in this study.

The patient appeared to have intact light touch sensation and proprioception, which suggested he did not have subluxation of the left shoulder. He reported independence in activities of daily living, and there was an absence of unilateral neglect, apraxia, and sensory disturbance of the affected UE. He had previously received conventional physical therapy such as gentle massage, range of motion exercises, stretching, and strengthening exercises. On screening, he had active shoulder flexion to $50^{\circ}$, elbow extension to $70^{\circ}$, and wrist extension of $10^{\circ}$ from full flexion. However, he was unable to extend any digits of his affected hand. There were no significant visual or cognitive deficits ( 30 points on the Mini-Mental Status Examination). The participant signed an informed consent for participation in this study, and a community-based Institutional Review Board approved the protocol.

The FIST approach required the subject to partially support his body weight with his UE on a treadmill customized with suspension frames and a harness apparatus combined with a functional electric stimulator. The treadmill speed was set between $0.3 \mathrm{~km} / \mathrm{h}$ and $2 \mathrm{~km} / \mathrm{h}$, which was sufficient to initiate stepping with the patient's arms ${ }^{10}$. FES was used to activate the extensor carpi radialis (ECR) and triceps brachii (TRI) so that adequate joint movement (throughout the possible range of motion), at the appropriate time, could be elicited. This allowed normal arm movement with swing phase flexion and stance phase extension patterns to be produced with repetitive practice. The specific parameters of the FES were as follows: i) frequency $=20-40 \mathrm{~Hz}$; ii) pulse width $=200-400 \mathrm{~ms}$; iii) amplitude=biphasic waveform of 20-50 mA; and iv) an on/off ratio of $1: 1^{11)}$. The FES was discontinued after 3 weeks, at which point the patient was able to fully extend his elbow independently.

The patient lay with his abdomen on the table. His arms, including his shoulder, elbow and wrist, were fully exposed to the treadmill against gravity. His body weight was partially supported by the table during the treadmill training. The patient was instructed to attain $130^{\circ}$ of shoulder flexion in the involved upper limb, with the elbow and wrist maximally extended, during the swing phase. During the stance phase in the prone position, to ensure that the elbow, the palmar surface of the hand, and the metacarpal heads were adequately loaded, the patient was instructed to maintain $60^{\circ}$ of shoulder flexion. This forced intensive hand weight bearing during the arm stepping movements was designed to facilitate rhythmic flexion and extension patterns of the elbow and wrist extensors, thus resulting in muscle strengthening. The therapist guided the extension of the patient's elbow and wrist using verbal and tactile feedback and encouragement, along with sensorimotor stimulation of the UE via an approximation technique, as needed. However, as the patient was able to produce elbow extension during the stance phase and wrist extension during the swing phase on his own, which consistently occurred after 5 sessions, verbal and FES-initiated proprioceptive stimuli were gradually withdrawn. The intervention lasted 60 minutes (10 minutes of exercise, 2 minutes of rest) a day, 5 times a week for 6 weeks.

An experienced radiologist performed the USI scanning. The experimenter responsible for assessing the outcomes was unaware of who the subject was and the level of treatment. Pre- and post-intervention tests included USI and standardized UE motor function tests. The modified Ashworth scale (MAS), which is a reliable tool for analyzing elbow flexor muscles, was used to measure spasticity ${ }^{12)}$.

A 'LOGIQ sonography system ( $\alpha$ 200, Samsung-GE Medical Systems Co., Ltd., Seongnam, Republic of Krorea)' with a 7.5-MHz linear transducer was used with ultrasound gel (AQUASONIC ${ }^{\circledR} 100$, Parker Inc., Orange, NJ, USA) on the transducer head. For USI of the ECR muscle, the patient was positioned supine with the elbow fully flexed $\left(180^{\circ}\right)$ and the forearm pronated $90^{\circ}$. The patient was instructed to relax all of the muscles, and a reference point was used to mark the head of the radius. To check the anatomical landmark, USI of the ECR was first performed located longitudinally over the marked reference point. The transducer head was then maneuvered distally $2 \mathrm{~cm}$ from the head of the radius and rotated $90^{\circ}$ to transversally align the head with the muscles until the sharpest cross-sectional area (CSA) of the complete ECR was identified through visual inspection during USI. The full CSA of the ECR was examined during resting and contraction, and an automatic monitor caliper was used to trace around the ECR muscle border.

For USI of the TRI muscle, the subject was positioned prone with the head directed to the side being measured and the shoulder abducted and elbow flexed at $90^{\circ}$. The limb was supported on a cushion and was vertically hung over the side of the measurement table. The anatomical landmark used for the TRI was the olecranon process of the ulna, and USI was performed longitudinally explored with the TRI in a relaxed position. The transducer head was rotated $90^{\circ}$ and moved proximally $10 \mathrm{~cm}$ from the olecranon process until the sharpest CSA of the whole TRI was visible and measured. An image of the CSA image of the TRI muscle was captured during both the resting and contracting states. Screen calipers were used to trace around the muscle border. An immediate muscle dimension (i.e., CSA) was displayed and stored, and the means of two of three scans performed on both sides were calculated for analysis. The scanning was repeated until clear acceptable measurements were obtained.

The manual function test (MFT), Fugl-Meyer assessment (FMA), and Jebsen-Taylor hand function test (JTHFT) were used in this case study. These are well-designed, feasible, and efficient clinical examination methods that have been tested to determine functional gross and fine motor skills in the affected extremity in patient with stroke.

The MFT, whose reliability is well established ${ }^{13)}$, is composed of 32 test elements that examine arm motion and manipulative factors. Arm motion factors consist of forward 
Table 1. CSA from ultrasound imaging in the both extensor carpi radialis and triceps brachii muscle before (preFIST) after (post FIST) the intervention

\begin{tabular}{llcccccc}
\hline & & \multicolumn{2}{c}{ Extensor carpi radialis $\left(\mathrm{cm}^{2}\right)$} & \multicolumn{3}{c}{ Triceps brachii $\left(\mathrm{cm}^{2}\right)$} \\
\hline & & Rest & Contraction & $\begin{array}{c}\text { Contraction- } \\
\text { Rest }\end{array}$ & Rest & \multicolumn{2}{c}{$\begin{array}{c}\text { Contraction } \\
\text { Contraction- } \\
\text { Rest }\end{array}$} \\
\hline \multirow{2}{*}{ Pre-FIST } & Rt & 3.21 & 3.86 & 0.65 & 7.50 & 8.32 & 0.82 \\
& Lt & 2.47 & 2.75 & 0.28 & 6.75 & 7.05 & 0.30 \\
\multirow{2}{*}{ Post-FIST } & Rt & 3.34 & 4.10 & 0.76 & 7.67 & 8.65 & 0.98 \\
& Lt & 3.12 & 3.75 & 0.63 & 7.35 & 8.25 & 0.90 \\
\multirow{2}{*}{ Improvement (\%) } & Rt & 3.89 & 5.85 & 14.47 & 2.22 & 3.82 & 16.33 \\
& Lt & 20.83 & 26.67 & 55.56 & 8.16 & 14.55 & 66.67 \\
\hline
\end{tabular}

FIST: forced intensive strengthening technique

Table 2. Motor function test before (pre-FIST) and after (post-FIST) the FIST

\begin{tabular}{lccccccccc}
\hline & $\begin{array}{c}\text { MAS } \\
\text { (score) }\end{array}$ & \multicolumn{2}{c}{$\begin{array}{c}\text { Dynamometer } \\
(\mathrm{kg})\end{array}$} & \multicolumn{2}{c}{$\begin{array}{c}\text { MFT } \\
\text { (score) }\end{array}$} & \multicolumn{2}{c}{$\begin{array}{c}\text { FMA } \\
\text { (score) }\end{array}$} & \multicolumn{2}{c}{$\begin{array}{c}\text { JTHFT } \\
\text { (seconds) }\end{array}$} \\
\hline & & $\mathrm{Rt}$ & $\mathrm{Lt}$ & $\mathrm{Rt}$ & $\mathrm{Lt}$ & $\mathrm{Rt}$ & $\mathrm{Lt}$ & $\mathrm{Rt}$ & $\mathrm{Lt}$ \\
Pre-FIST & $1+$ & 36 & 15 & $27 / 32$ & $16 / 32$ & $52 / 66$ & $29 / 66$ & $20 / 60$ & $38 / 60$ \\
Post-FIST & 1 & 41 & 32 & $30 / 32$ & $27 / 32$ & $60 / 66$ & $49 / 66$ & $13 / 60$ & $19 / 60$ \\
Improvement (\%) & 12 & 53 & 9 & 34 & 12 & 30 & 12 & 32 & \\
\hline
\end{tabular}

FIST: forced intensive strengthening technique; MAS: modified Ashworth scale; MFT: manual function test; FMA: Fugl-Meyer assessment; JTHFT: Jebsen-Taylor hand function test

and lateral elevation of the UE by touching the occiput and the dorsum of the palm. Manipulative factors consist of grasping, pinching, carrying a cube, and the Purdue pegboard. The MFT measures the maximum performance rather than the average performance, and each item is scored either failure $(0)$ or success $(1)^{13)}$. The UE motor subscore of the FMA, which tests the motor skill, coordination, and speed of the UE and ranges from 0 to 66 points, was used in this study. The reliability and validity of the FMA has been confirmed in previous studies ${ }^{14)}$. The JTHFT is widely used for assessing activities of daily living in neuro-rehabilitation settings ${ }^{15)}$; it measures the time required to complete 7 different UE tasks sequentially as accurately and quickly as possible ${ }^{15)}$. Stern, 1992 E.B. Stern, Stability of the Jebsen-Taylor hand function test across three test sessions, Am. J. Occup. Ther. 46 (1992), pp. 647-649. View Record in Scopus | Cited By in Scopus (19). The reliability and validity of JTHFT are good, and the test is reliable in a variety of populations ${ }^{15)}$.

\section{RESULTS}

Table 1 shows the CSAs of the ECR and TRI during relaxation and contraction, as well as the difference between the relaxation and contraction states before and after the FIST intervention. The actual FIST-induced improvement (\%) was determined as (CSA Contract - CSA Rest)/CSA Contract $\times 100$. The difference between relaxation and contraction of the affected ECR muscle increased from 0.28 to $0.63 \mathrm{~cm}^{2}$ (55.56\% improvement), while that of the affected TRI muscle increased from 0.30 to $0.90 \mathrm{~cm}^{2}(66.67 \% \mathrm{im}-$ provement).

The MAS score of the affected elbow flexor muscle was reduced from $1+$ to 1 after the intervention. Muscle strength, as measured with a handheld dynamometer, of the affected UE increased from 15 to $32 \mathrm{~kg}$ (53\% improvement). The MFT score of the affected UE also increased from 16/32 to 27/32 (34\% improvement). Furthermore, the FMA score of the affected UE rose from $29 / 66$ to $49 / 66$ (30\% improvement). On the other hand, the mean time in the JTHFT for the affected UE decreased from $38 / 60$ to $19 / 60 \mathrm{sec}(32 \%$ improvement). These findings indicate that the FIST intervention improved muscle power and motor functional skill (Table 2).

\section{DISCUSSION}

Our data show that the FIST intervention was effective in increasing functional motor performance and muscle size of the UE in a hemiparetic stroke patient. Functional motor performance associated with strengthening of the UE is a key contributor to improved ability to perform activities of daily living in patient with stroke ${ }^{1)}$. Also, clinical measures such as the MFT, FMA, and JHTFT and the size of the ECR and TRI muscles have been considered critical aspects in demonstrating recovery of UE function in stroke patients. Although this is a pilot study, we were able to demonstrate a clinical trial highlighting the effect of the FIST on functional motor performance and associated muscle strengthening along with subjective satisfaction in a patient with stroke.

Although the FIST intervention had previously only been used in a child with cerebral palsy ${ }^{16)}$, it was adequate for applying appropriate UE loading in the adult patient presented here. Strengthening exercises combined with FES have been reported as being able to improve UE function in stroke 
patient ${ }^{6,8)}$. However, this is the first study to report the combined effect of supported treadmill strengthening exercises and FES in an adult patient with stroke.

During the FIST intervention, the patient, who originally had clumsy hand function, gradually developed an appropriate sequential response and a reciprocal stepping movement of the UE. This reciprocal stepping movement may help with the transition to actual activities of daily living, as well as strengthening of the UE. Furthermore, in the current study, the participant realized full movement of his trunk on the table while in a prone position with his neck exposed and his head under the influence of gravity on the edge of the table. Therefore, exposure to gravity could be limited to his breathing pattern, and UE stepping movement, thus reducing the transmission of loading to the head.

Our USI data demonstrated that the sizes of the ECR and TRI were substantially enhanced in response to the FIST intervention. According to the USI results, the FIST intervention proved to be an effective technique for initiating musculoskeletal changes in the patient, and this might be a sign of intensive strengthening in the chronic stroke patient. Although it was difficult to compare our results with results of past research, Lee et al. ${ }^{16)}$ noted an increase in the CSAs of the ECR ( 0.20 to $0.30 \mathrm{~cm}^{2} ; 39 \%$ improvement) and TRI ( 0.15 to $0.46 \mathrm{~cm}^{2} ; 67 \%$ improvement). In the present study, the CSA of the ECR ( 0.28 to $0.63 \mathrm{~cm}^{2} ; 56 \%$ improvement) and TRI ( 0.30 to $0.90 \mathrm{~cm}^{2} ; 67 \%$ improvement) muscles were also found to have increased. Furthermore, intensive and repetitive use of the UE combined with partial weight-bearing on a treadmill may assist the patients in reducing adaptations in the chronic stroke stage and thus help them improve their motor function.

It is our clinical opinion that this study shows the benefits of the FIST in a clinical and quantitative way. This work also highlights that the FIST may have the advantageous effects on the motor functions associated with activities of daily living of the upper extremity in a stroke patient. UE functional performance was measured with the MFT, FMA, and JTHFT. The MFT reflects the level of impaired motor function of the affected UE, and changes in the MFT during the rehabilitation process will correspond with how much the patient has recovered, specifically, the relationship between the duration of onset and the level of impaired motor function ${ }^{13)}$. The FMA is one of the most comprehensive quantitative measures of motor impairment following stroke, and it is recommended for clinical trials of stroke rehabilitation ${ }^{14)}$. The JTHFT is the most commonly used assessment of activities of daily living and can be completed quickly ${ }^{15}$. Based on these advantages, the MFT, FMA, and JTHFT were selected for this study.

The effectiveness of the FIST intervention in improving UE function in the stroke patient is evident in the clinical measurements. The MFT score of the affected limb showed a 34\% improvement (from 16 to 27). Specifically, fine motor performance increased with gross motor performance, as hand mobility (fine motor performance) may be improved following by shoulder stability (gross motor performance). The FMA scored showed a 20-point gain (from 29 to 49), and these differences reached a clinically meaningful value $^{14)}$. Specially, there was a remarkable increase in ac- tive shoulder and elbow flexion ROM in the FMA analysis. Movement time of the functional arm and hand motor tasks, as measured with the JTHFT, also improved. It is clear that the movement time of the UE plays a role in shaping functional neuromuscular control in motor skill learning during rehabilitation. Previous studies have also shown that the recovery of UE function in stroke patients was not enhanced by task-oriented training alone but required repetitive task-oriented training associated with different interventions ${ }^{5,10,16)}$. In other words, repeated use of the affected UE is one of the key elements for functional restoration in stroke patients. Most of all, in this study, performance of isolated arm movements was improved with better control of coordinated multi-joint movements although the patient did not regain full function.

This study had a few limitations that need to be addressed. First, it used only a single participant, and this will need to be increased in the future to improve clinical relevance. Second, the absence of a control group inevitably presented difficulties that influenced the reliability and validity of the results of this study. Third, this report represents a preliminary experiment intended to investigate the short-term effect of the FIST protocol in a stroke patient. Thus, at this time, the technique discussed here cannot be said to provide an optimal strategy for training UE muscle control. Nevertheless, our findings regarding this strength technique make an important contribution to the existing body of knowledge related to therapeutic exercises for the patients with hemiplegia stroke in whom current strengthening exercises are not easily applicable due to severe impairments such as pain and weakness.

\section{ACKNOWLEDGEMENT}

This work was supported by Business for Cooperative R\&D between Industry, Academy, and Research Institutes funded by the Korea Small and Medium Business Administration in 2014 (Grant Number C0212258).

\section{REFERENCES}

1) Fregni F, Pascual-Leone A: Hand motor recovery after stroke: tuning the orchestra to improve hand motor function. Cogn Behav Neurol, 2006, 19: 21-33. [Medline] [CrossRef]

2) Bohannon RW: Adequacy of simple measures for characterizing impairment in upper limb strength following stroke. Percept Mot Skills, 2004, 99: 813-817. [Medline]

3) Page SJ, Levine P, Sisto $S$, et al.: Stroke patients' and therapists' opinions of constraint-induced movement therapy. Clin Rehabil, 2002, 16: 55-60. [Medline] [CrossRef]

4) Hummelsheim H, Hauptmann B, Neumann S: Influence of physiotherapeutic facilitation techniques on motor evoked potentials in centrally paretic hand extensor muscles. Electroencephalogr Clin Neurophysiol, 1995, 97: 18-28. [Medline] [CrossRef]

5) van der Lee JH, Wagenaar RC, Lankhorst GJ, et al.: Forced use of the upper extremity in chronic stroke patients: results from a single-blind randomized clinical trial. Stroke, 1999, 30: 2369-2375. [Medline] [CrossRef]

6) Bütefisch C, Hummelsheim H, Denzler P, et al.: Repetitive training of isolated movements improves the outcome of motor rehabilitation of the centrally paretic hand. J Neurol Sci, 1995, 130: 59-68. [Medline] [CrossRef]

7) Renner CI, Woldag H, Atanasova R, et al.: Change of facilitation during voluntary bilateral hand activation after stroke. J Neurol Sci, 2005, 239: 25-30. [Medline] [CrossRef]

8) Faghri PD, Rodgers MM, Glaser RM, et al.: The effects of functional 
electrical stimulation on shoulder subluxation, arm function recovery, and shoulder pain in hemiplegic stroke patients. Arch Phys Med Rehabil, 1994 75: 73-79. [Medline]

9) Rezaoltani A: The applicability of muscle ultrasonography in physiotherapy researches. J Phys Ther Sci, 2003, 15: 33-37. [CrossRef]

10) Daly JJ, Ruff RL: Feasibility of combining multi-channel functional neuromuscular stimulation with weight-supported treadmill training. J Neurol Sci, 2004, 225: 105-115. [Medline] [CrossRef]

11) Cui BJ, Wang DQ, Qiu JQ, et al.: Effects of a 12-hour neuromuscular electrical stimulation treatment program on the recovery of upper extremity function in sub-acute stroke patients: a randomized controlled pilot trial. J Phys Ther Sci, 2015, 27: 2327-2331. [Medline] [CrossRef]

12) Bohannon RW, Smith MB: Interrater reliability of a modified Ashworth scale of muscle spasticity. Phys Ther, 1987, 67: 206-207. [Medline]
13) Miyamoto S, Kondo T, Suzukamo $Y$, et al.: Reliability and validity of the Manual Function Test in patients with stroke. Am J Phys Med Rehabil, 2009, 88: 247-255. [Medline] [CrossRef]

14) Sanford J, Moreland J, Swanson LR, et al.: Reliability of the Fugl-Meyer assessment for testing motor performance in patients following stroke. Phys Ther, 1993, 73: 447-454. [Medline]

15) Hackel ME, Wolfe GA, Bang SM, et al.: Changes in hand function in the aging adult as determined by the Jebsen Test of Hand Function. Phys Ther, 1992, 72: 373-377. [Medline]

16) Lee DR, You JH, Lee NG, et al.: Comprehensive Hand Repetitive Intensive Strengthening Training (CHRIST)-induced morphological changes in muscle size and associated motor improvement in a child with cerebral palsy: an experimenter-blind study. NeuroRehabilitation, 2009, 24: 109-117. [Medline] 